Published in final edited form as:

Nano Lett. 2008 December ; 8(12): 4386-4390. doi:10.1021/n1802245w.

\title{
Aptamer-based SERRS Sensor for Thrombin Detection
}

\author{
Hansang Cho ${ }^{1,2,3}$, Brian R. Baker ${ }^{2}$, Sebastian Wachsmann-Hogiu ${ }^{3}$, Cynthia V. Pagba ${ }^{3}$, Ted $^{2}$ \\ A. Laurence ${ }^{2}$, Stephen M. Lane ${ }^{2,3}$, Luke P. Lee ${ }^{1, *}$, and Jeffrey B.-H. Tok ${ }^{2,3,{ }^{*}}$ \\ ${ }^{1}$ Biomolecular Nanotechnology Center, Berkeley Sensor \& Actuator Center, Department of \\ Bioengineering, University of California, Berkeley, CA 94720 \\ ${ }^{2}$ Lawrence Livermore National Laboratory, Livermore, CA 94550 \\ ${ }^{3}$ NSF Center for Biophotonics, University of California, Davis, CA 95817
}

\begin{abstract}
We describe an aptamer-based Surface Enhanced Resonance Raman Scattering (SERRS) sensor with high sensitivity, specificity, and stability for the detection of a coagulation protein, human athrombin. The sensor achieves high sensitivity and a limit of detection of $100 \mathrm{pM}$ by monitoring the SERRS signal change upon the single step of thrombin binding to immobilized thrombin binding aptamer. The selectivity of the sensor is demonstrated by the specific discrimination of thrombin from other protein analytes. The specific recognition and binding of thrombin by the thrombin binding aptamer is essential to the mechanism of the aptamer-based sensor, as shown through measurements using negative control oligonucleotides. In addition, the sensor can detect 1 $\mathrm{nM}$ thrombin in the presence of complex biofluids, such as $10 \%$ fetal calf serum, demonstrating that the immobilized, 5'-capped, 3'-capped aptamer is sufficiently robust for clinical diagnostic applications. Furthermore, the proposed sensor may be implemented for multiplexed detection using different aptamer-Raman probe complexes.
\end{abstract}

\section{Keywords}

aptamer; oligonucleotide; aptasensor; nanosensor; surface-enhanced resonance Raman spectroscopy; thrombin detection

\begin{abstract}
As nanobiotechnology progresses, aptamers, single-stranded (ss) oligonucleotides ( $<100 \mathrm{nt}$ ), have been promoted as ideal diagnostic reagents and potential antibody replacements for the development of biomolecular nanosensors due to their high affinity, specificity, and stability. ${ }^{1-9}$ Interestingly, certain aptamers have been reported to undergo distinguishable conformational changes upon interaction with their targets. ${ }^{10-15}$ Recently, numerous aptamer-based sensors (aptasensors or aptamer beacons) have exploited binding-induced conformational changes to monitor the interaction with targets by measuring electron transfer ${ }^{10,11,16,17}$, color change ${ }^{12-14,18}$, or fluorescence quenching ${ }^{15,19,20}$. However, these methods are limited by either the size or sensitivity of the sensors or by the complexity of the assays involved. For example, electrochemical sensors designed to measure electron transfer require electrodes on the millimeter scale to achieve sufficient sensitivity;
\end{abstract}

\footnotetext{
*To whom correspondence should be addressed. Current Address Jeffrey B.-H. Tok, Ph.D., Micropoint Biosciences Inc., 1250 Oakmead Parkway, Suite 107, Sunnyvale, CA 94085, (408) 732-5588, jeff.tok@ micropointbio.com, Professor Luke P. Lee, Biomolecular Nanotechnology Center, Berkeley Sensor \& Actuator Center, Department of Bioengineering, University of California, Berkeley, 408C Stanley Hall, Berkeley, CA 94720, (510) 642-5855, lplee@ berkeley.edu.

Supporting Information Available: materials, preparation of sensor, experimental conditions, and evaluation results on $50 \%$ signal saturation. This material is available free of charge via the Internet at http://pubs.acs.org.
} 
colorimetric methods require a large amount of particle aggregation to induce a discernable color change; the previously reported surface-enhanced Raman scattering (SERS) aptasensor used multiple binding events, a sandwich assay requiring labeling of target molecules with the complex of gold nanoparticle/aptamer/Raman probe/silver nanoparticle after capturing the target. ${ }^{21,22}$

Herein, we report a sensitive, selective, and stable aptamer-based SERRS sensor for a coagulation protein, human a-thrombin. The sensing mechanism is based on the single-step target binding event to aptamer, which results in a decrease of the intensity of SERRS signals of the probe molecule attached to thrombin binding aptamer (TBA) due to the displacement of TBA from a gold nanoparticle (GNP) surface. We hypothesize that the thrombin molecules, after dissociating from the displaced TBA, are able to participate in further binding/displacement event with TBA immobilized on the GNP surface. Furthermore, the SERRS technique provides ultra sensitive detection of the probe molecule by combining resonance Raman scattering with SERS enhancement in the presence of a metallic nanoparticles. As a result, our described aptamer-based SERRS sensor allows much simpler detection scheme, coupled with a lower limit of detection when compared to other techniques based on multiple binding events or non-resonant SERS.

TBA displacement event is realized by exploiting the propensity of single-stranded DNA (ssDNA) to readily absorb onto GNP surfaces and to subsequently desorb upon its target binding. ${ }^{12,13,23,24}$ For the present study, a 15-mer TBA (5'-GGT TGG TGT GGT TGG-3' "G" marked in bold is essential for quadruplex formation for thrombin binding) previously reported to bind thrombin with a dissociation constant of $25 \mathrm{nM}$, was used. ${ }^{25,26}$ A thiol functional group was added to the $5^{\prime}$ end for covalent binding to the gold surface, and the Raman probe (methylene blue, MB) was covalently bound to the $3^{\prime}$ end. The full sequence is as follows: [ $5^{\prime}-\left(\mathrm{CH}_{2}\right)_{6}$-S-S- $\left(\mathrm{CH}_{2}\right)_{6}$-TAA GTT CAT CTC CCC GGT TGG TGT GGT TGG T-MB-3']. The thiolized TBA is adsorbed on the surface in two populations: chemical and physical adsorption. ${ }^{27,}{ }^{28}$ Chemisorbed TBA, bound through a covalent gold-thiol bond, would remain immobilized upon addition of sample solutions and provide a baseline signal level to verify the stability of the ssDNA aptamer and the light-sensitive MB during exposure to complex biofluids, surface plasmon heating, and incident laser. Physisorbed TBA, in contrast, is held to the GNP surface only through electrostatic interactions between nitrogenous bases and the GNP surface and will be susceptible to displacement from the surface upon recognition and binding of target molecules. $23,28,29$

Quantitative and sensitive detection of thrombin is achieved by monitoring the strong SERRS peaks of methylene blue, covalently attached to TBA. Methylene blue has a $S_{0} \rightarrow S_{1}$ transition centered at around $660 \mathrm{~nm}$, allowing the incoming light in $632 \mathrm{~nm}$ wavelength to excite resonant vibration of the molecule. As a result, SERRS enhances Raman scattering signals by $10^{3}-10^{5}$ times compared with non-resonant SERS while providing narrow spectral lines suited to quantitative analysis. ${ }^{30,31}$

Our detection mechanism is based on the change of SERRS intensity of the Raman probe (Fig. 1). In the absence of thrombin, single-stranded TBA is unfolded and adsorbed onto a GNP surface. This brings the methylene blue in close proximity to the GNP surface (Fig. 1a), resulting in surface enhanced Raman scattering for methylene blue. Upon introduction of the target protein, TBA undergoes a conformational change (G-quadruplex formation) induced by a single-step binding event with thrombin (Fig. 1b). TBA is thus being displaced from the GNP surface, hence reducing the intensity of the SERRS signal for MB.

The aptamer-based SERRS sensor was fabricated by a simple two-step procedure (see supporting information for a detailed description). Briefly, $80 \mathrm{~nm}$ colloidal GNPs were first 
aggregated on a glass cover slip previously treated with 3-aminopropyltriethoxysilane (APTES) (Fig. 1c). $\mathrm{CuSO}_{4}$ was used as an aggregation agent, since the charge on $\mathrm{Cu}^{2+}$ induces aggregation of GNP covered with halide anions resulting in a fractal structure of GNPs. ${ }^{32-34}$ The aggregated GNPs presumably provide hot spots, where a strong surface plasmon is resonated locally. The APTES-coated glass retains GNPs on the surface through harsh rinsing steps and also prevents any further aggregation of GNPs that may be caused by salt ions. Secondly, MB-conjugated TBA was introduced to the GNP-aggregated substrate at room temperature and allowed to incubate overnight in the dark (Fig. 1d). The glass slide was then thoroughly rinsed with $1 \mathrm{x}$ thrombin binding buffer (TBB) and the SERRS spectra were collected. Finally thrombin was introduced and incubated for one hour at room temperature in the dark (Fig. 1e). The substrate was rinsed, kept in 1x TBB solution and covered with a cover glass.

The spectra were collected from aggregated GNPs in a fractal structure (Fig. 2 inset, SEM image). The whole spectra are displayed after subtracting the fluorescence background from the original signal (Fig. 2a) for clarity and the most prominent Raman peak at $1622 \mathrm{~cm}^{-1}$ (assigned to $v(\mathrm{CC})$ ring and $v(\mathrm{CNC})$ ring modes of methylene blue) ${ }^{35}$ was compared for quantitative analysis (Fig. 2b, 2c). The effect of adding thrombin to the aptamer can clearly be observed through the reduction of the peak's intensity at the $1622 \mathrm{~cm}^{-1}$. Initially, the peak's intensity dropped approximately $10 \%$ when introduced with $100 \mathrm{pM}$ thrombin. However, with $\geq 1 \mu \mathrm{M}$ thrombin concentration, the intensity dropped to about $50 \%$, the observed saturation level (Fig. 2c). We thus estimate the limit of detection for this assay to be $100 \mathrm{pM}$ with a dynamic range spanning $100 \mathrm{pM}$ to $1 \mu \mathrm{M}$.

To verify that the signal change is dependent on the specific recognition of thrombin, we added a nonspecific binding protein, bovine serum albumin (BSA). When the substrate was treated with increasing concentration of BSA (up to $1 \mu \mathrm{M}$ BSA), we did not observe any significant change in the SERRS signal (Fig. 2c). This suggests that the TBA was not displaced from the GNP substrate, as TBA did not bind to BSA. The specificity of the aptamer-based SERRS method was further investigated with a mutant TBA $\left[5^{\prime}-\left(\mathrm{CH}_{2}\right)_{6}-\mathrm{S}-\mathrm{S}\right.$ $\left(\mathrm{CH}_{2}\right)_{6}$-TAA GTT CAT CTC CCC AAT TGG TGT GGT TAA T-MB-3'] (nucleotides marked in bold indicate the position of essential " $G$ " in the original aptamer sequence) in $1 x$ TBB (Fig. 3). Upon the addition of $100 \mathrm{nM}$ thrombin to the mutant TBA-GNP substrate, we did not observe any decrease in the SERRS signal intensity. Taken together, these observations suggest that the displacement mechanism relies on specific recognition and that the aptamer-based SERRS method is highly specific for the detection of TBA-thrombin interaction.

The stability of the sensor was evaluated in the presence of complex mixtures including nucleases, i.e. serum (Fig. 4). We observed $\sim 20 \%$ signal decrease when the substrate was treated with $10 \%$ fetal calf serum $(v / v$ in TBB), indicating that the TBA was minimally displaced from the aggregated GNPs by the serum's constituents; the SERRS signal drop reached the $50 \%$ saturation in $75 \%$ serum. We hypothesize that the displacement of TBA in elevated serum levels was probably due to stronger electrostatic interference by serum's salt contents rather than by nuclease activity in the serum, which are unlikely to degrade the immobilized, 5'- and, 3'-capped TBA. ${ }^{36}$ With this "serum baseline," the intensity in the presence of $10 \%$ serum, we proceed to introduce thrombin to both the TBA-GNP substrate and the mutant TBA-GNP substrate. With the addition of $1 \mathrm{nM}$ thrombin in $10 \%$ serum to the TBA-GNP substrate, the signal dropped to $\sim 75 \%$ from the serum baseline, and the signal further decreased to a saturating level of $60 \%$ when the thrombin concentration increased to $100 \mathrm{nM}$. In contrast, the mutant TBA-GNP substrate did not produce any significant signal change when exposed to $1 \mathrm{nM}$ thrombin in $10 \%$ serum. These observations collectively indicate that the sensor can be performed in the presence of $10 \%$ 
serum. We also note that mutant TBA-GNP substrate is unaffected by nucleases in serum. Thus, the mutant aptamer both effectively and conveniently provides a "negative control" that enables specific protein detection for each respective assay performed in $10 \%$ serum.

The 50\% saturation level can be attributed to the chemisorbed aptamer population. To verify that the signal was stemming only from chemisorbed aptamer, we repeatedly subjected the substrate to harsh conditions: high $\mathrm{NaCl}$ (1.4 M), high serum (100\%) wash, and high thrombin $(1 \mu \mathrm{M})$ interaction (Fig. S1). In all cases we observed a 50\% maximum signal reduction, suggesting that $\sim 50 \%$ of the aptamer is chemisorbed onto the GNPs and is, therefore, resistant to displacement via disruption of electrostatic interactions rather than the denaturation of TBA.

In conclusion, we have demonstrated a highly sensitive, selective, and stable aptamer-based SERRS sensor for the detection of thrombin. The limit of detection is $100 \mathrm{pM}$ (below the dissociation constant of TBA, $25 \mathrm{nM}$ ). This low limit of detection is possible because the aptamer-based SERRS signal depends on the number of aptamers displaced from the surface, not the number of aptamers that are bound to target. In addition, this assay is sufficiently stable to identify the intended target in the presence of $10 \%$ serum. Given the size and simplicity of the proposed detection schematic, this method may be readily used in a miniaturized and integrated platform as it can easily accommodate numerous proteinspecific aptamers with a variety of Raman probes for high throughput and multiplexed drug screening and biomedical diagnostics.

\section{Supplementary Material}

Refer to Web version on PubMed Central for supplementary material.

\section{Acknowledgments}

This work has been supported by funding from Lawrence Livermore National Laboratory (LLNL-JRNL-405475) for a Lawrence Scholar Program predoctoral fellowship, Intel, Inc. (Integrated Confocal Nanofluidic SERS for Proteomics) and DARPA (HR0011-06-1-0050) for HC, the partial support of NIH Grant AI065359 for JBH, and the National Science Foundation Center for Biophotonics managed by UC Davis (PHY 0120999). Authors acknowledge Tammy Olsen for providing hollow gold nanoparticles, which were used for the preliminary experiments, Nicholas O. Fischer for providing guidance in aptamer immobilization.

\section{Abbreviations}

$\begin{array}{ll}\text { SERRS } & \text { surface-enhanced resonance Raman scattering } \\ \text { SERS } & \text { surface-enhanced Raman scattering } \\ \text { GNP } & \text { gold nanoparticle } \\ \text { TBA } & \text { thrombin binding aptamer } \\ \text { mutant TBA } & \text { non-thrombin binding oligonucleotide } \\ \text { sSDNA } & \text { single-stranded DNA } \\ \text { nt } & \text { nucleotides } \\ \text { MB } & \text { methylene blue } \\ \text { APTES } & \text { 3-Aminopropyltriethoxysilane } \\ \text { BSA } & \text { bovine serum albumin } \\ \text { TBB } & \text { thrombin binding buffer }\end{array}$



SEM
scanning electron microscope
LSPR
local surface plasmon resonance.

\section{REFERENCES}

1. Ellington AD, Szostak JW. Nature. 1990; 346(6287):818-822. [PubMed: 1697402]

2. Tuerk C, Gold L. Science. 1990; 249(4968):505-510. [PubMed: 2200121]

3. Jayasena SD. 1999; 45(9):1628-1650.

4. Sullenger BA, Gilboa E. Nature-review. 2002; 418(6894):252-258.

5. Pestourie C, Tavitian B, Duconge F. Biochimie. 2005; 87(9-10):921-930. [PubMed: 15963620]

6. Bunka DHJ, Stockley PG. Nat Rev Micro. 2006; 4(8):588-596.

7. Klussmann, S. The aptamer handbook: functional oligonucleotides and their applications. KGaA: WILEY-VCH Verlag GmbH \& Co; 2009.

8. Fischer NO, Tarasow TM, Tok JBH. Current Opinion in Chemical Biology. 2007; 11(3):316-328. [PubMed: 17548236]

9. Szakacs G, Paterson JK, Ludwig JA, Booth-Genthe C, Gottesman MM. Nat Rev Drug Discov. 2006; 5(3):219-234. [PubMed: 16518375]

10. Xiao Y, Piorek BD, Plaxco KW, Heeger AJJ. Am. Chem. Soc. 2005; 127(51):17990-17991.

11. Baker BR, Lai RY, Wood MS, Doctor EH, Heeger AJ, Plaxco KW. J. Am. Chem. Soc. 2006; 128(10):3138-3139. [PubMed: 16522082]

12. Stojanovic MN, Landry DW. J. Am. Chem. Soc. 2002; 124(33):9678-9679. [PubMed: 12175205]

13. Huang CC, Huang YF, Cao Z, Tan W, Chang HT. Anal. Chem. 2005; 77(17):5735-5741. [PubMed: 16131089]

14. Liu J, Lu Y. Nat. Protocols. 2006; 1(1):246-252.

15. Bagalkot V, Zhang L, Levy-Nissenbaum E, Jon S, Kantoff PW, Langer R, Farokhzad OC. Nano Lett. 2007; 7(10):3065-3070. [PubMed: 17854227]

16. Xiao Y, Lubin AA, Heeger AJ, Plaxco KW. Angewandte Chemie International Edition. 2005; 44(34):5456-5459.

17. Radi AE, AceroSanchez JL, Baldrich E, O'Sullivan CK. J. Am. Chem. Soc. 2006; 128(1):117-124. [PubMed: 16390138]

18. Wei H, Li B, Li J, Wang E, Dong S. Chemical Communications. 2007; (36):3735-3737. [PubMed: 17851611]

19. Fang X, Sen A, Vicens M, Tan W. ChemBioChem. 2003; 4(9):829-834. [PubMed: 12964156]

20. Shlyahovsky B, Li D, Weizmann Y, Nowarski R, Kotler M, Willner IJ. Am. Chem. Soc. 2007; 129(13):3814-3815.

21. Wang Y, Wei H, Li B, Ren W, Guo S, Dong S, Wang E. Chemical Communications. 2007; (48): 5220-5222. [PubMed: 18060148]

22. Chen J-W, Liu X-P, Feng K-J, Liang Y, Jiang J-H, Shen G-L, Yu R-Q. Biosensors and Bioelectronics. 2008; 24(1):66-71. [PubMed: 18436440]

23. Li H, Rothberg L. PNAS. 2004; 101(39):14036-14039. [PubMed: 15381774]

24. Li H, Rothberg LJ. Anal. Chem. 2004; 76(18):5414-5417. [PubMed: 15362900]

25. Bock LC, Griffin LC, Latham JA, Vermaas EH, Toole JJ. Nature. 1992; 355(6360):564-566. [PubMed: 1741036]

26. Vairamani M, Gross ML. J. Am. Chem. Soc. 2003; 125(1):42-43. [PubMed: 12515502]

27. Yau HCM, Chan HL, Sui Sf, Yang M. Thin Solid Films. 2002; 413:218-223.

28. Herne TM, Tarlov MJ. J. Am. Chem. Soc. 1997; 119(38):8916-8920.

29. Gearheart LA, Ploehn HJ, Murphy CJ. J. Phys. Chem. B. 2001; 105(50):12609-12615.

30. Nicolai SHA, Rubim JC. Langmuir. 2003; 19(10):4291-4294.

31. Stokes Robert J, Macaskill A, Lundahl PJ, Smith WE, Faulds K, Graham D. Small. 2007; 3(9): 1593-1601. [PubMed: 17647254] 
32. Bell SEJ, Sirimuthu NMS. J. Phys. Chem. 2005; 109(33):7405-7410.

33. Weitz DA, Oliveria M. Physical Review Letters. 1984; 52(16):1433.

34. Choi D, Kang TK, Cho H, Choi Y, Lee LP. Lab on a chip. 2008 In press.

35. Nicolai, SHdA; Rodrigues, PRP.; Agostinho, SML.; Rubim, JC. Journal of Electroanalytical Chemistry. 2002; 527(1-2):103-111.

36. Ruckman J, Green LS, Beeson J, Waugh S, Gillette WL, Henninger DD, Claesson-Welsh L, Janjic NJ. Biol. Chem. 1998; 273(32):20556-20567. 
a

b

TBA displacement

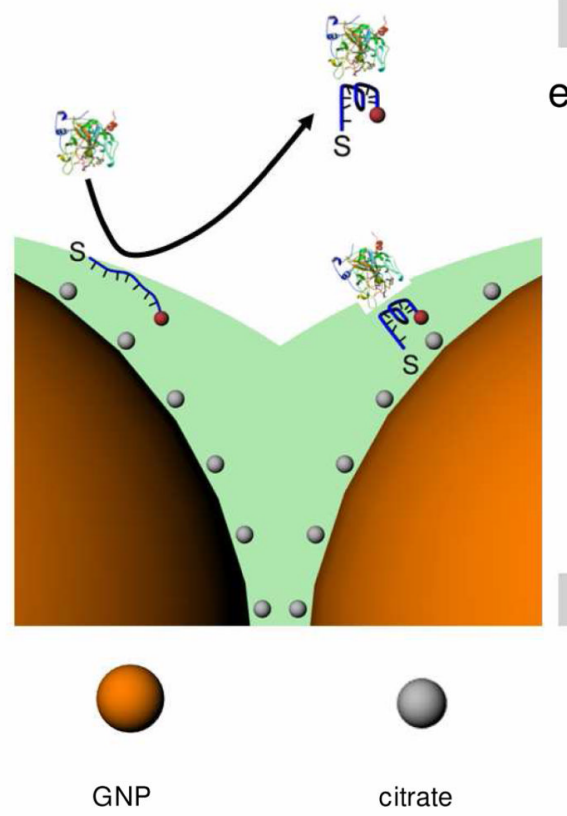

C

TBA adsorption

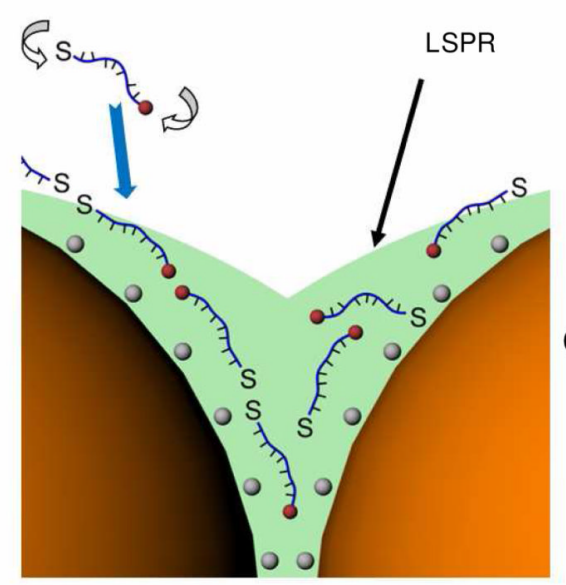

d

e

Figure 1.
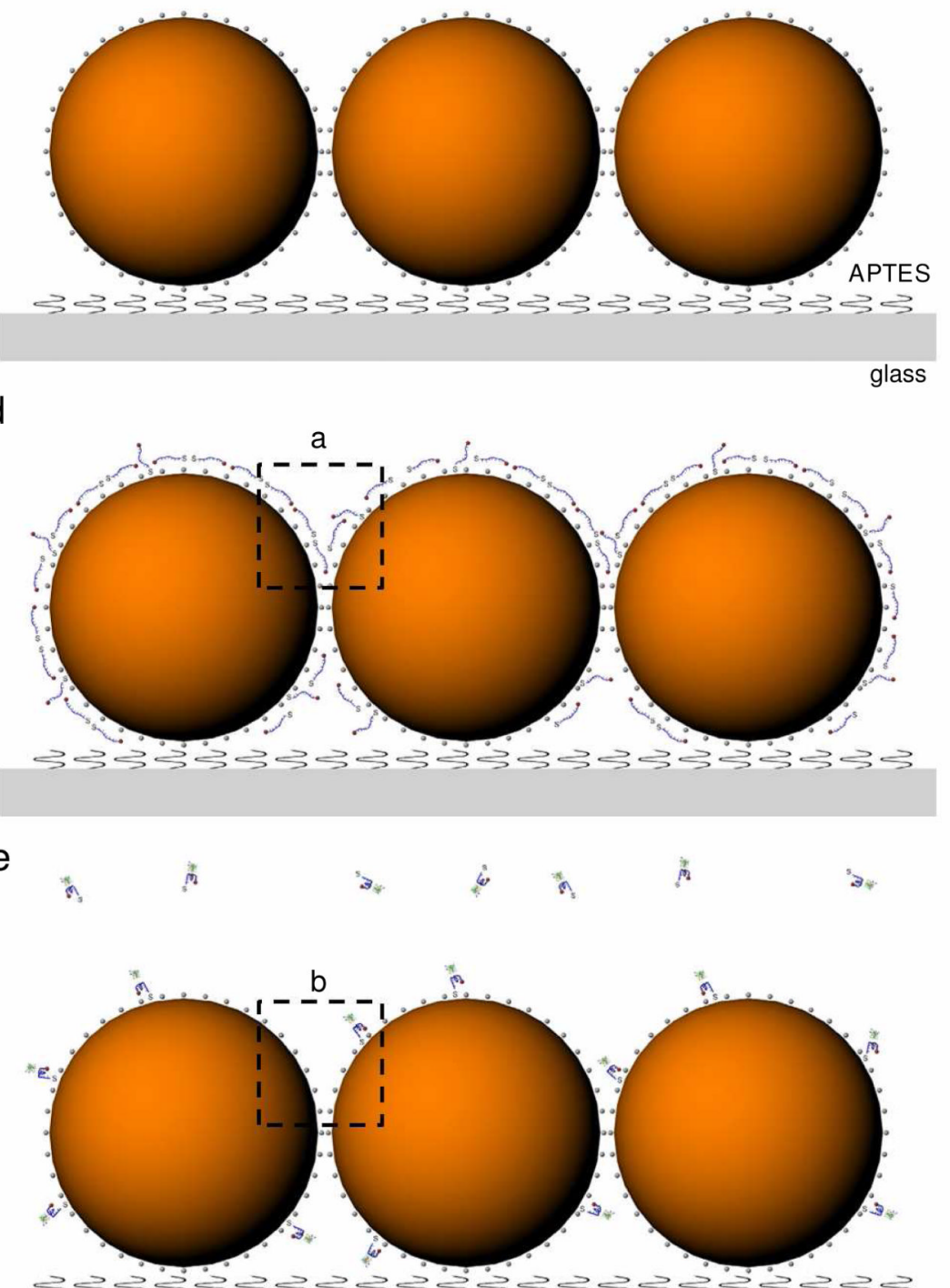

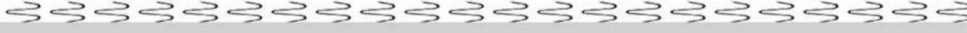

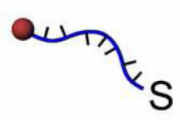

unfolded TBA with $\mathrm{MB}$

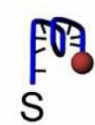

folded TBA

with $\mathrm{MB}$

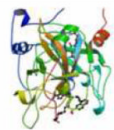

Human $\alpha$-thrombin

Schematics of detection method and preparation steps for the aptamer-based SERRS sensor. (a) A thiol-modified single-stranded TBA attached to MB, is naturally unfolded in the absence of human a-thrombin target molecule. The unfolded TBA is physisorbed on GNP surface through electrostatic interactions or chemisorbed through gold-thiol bonds. As a large number of MB is present in the hot spots, where local surface plasmon resonance is induced, a strong SERRS signal for MB is observed. (b) In the presence of thrombin, TBA changes conformation into a G-quadruplex form and physisorbed TBA is thus displaced from the surface, resulting in the prompt decrease of the SERRS signal. (c) GNP-substrate is prepared by aggregating citrate-coated GNPs on an APTES-treated glass substrate. (d) 
TBAGNP substrate is prepared by immobilizing MB-conjugated TBA on the GNP-substrate followed by a rinsing step removing unbounded TBA. (e) Thrombin is incubated on the prepared TBA-GNP substrate followed by a rinsing step removing displaced TBA-thrombin complex. 

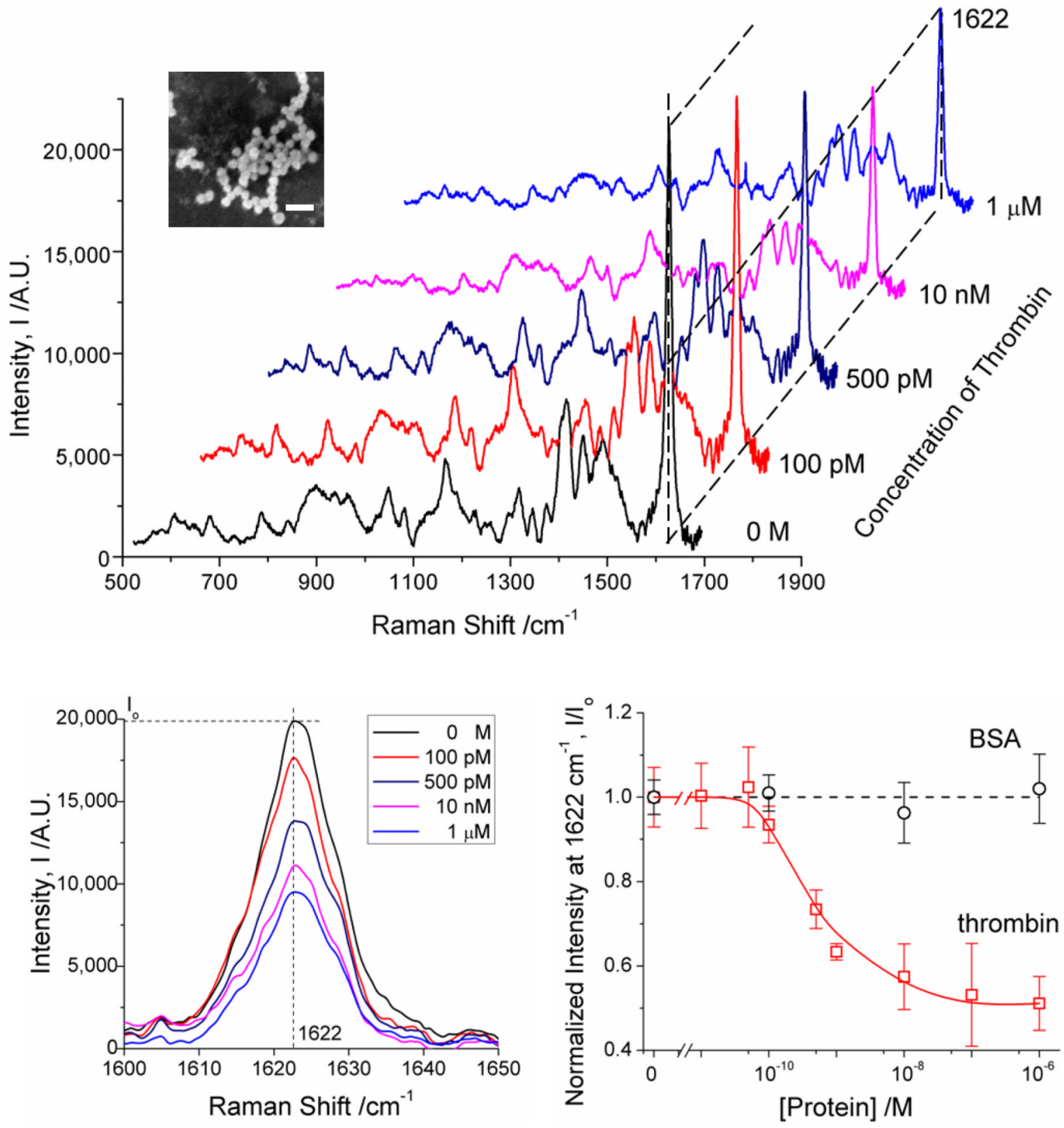

a

Figure 2.

Thrombin is quantitatively detected by measuring the SERRS signal drop for MB conjugated with TBA. (a) The SERRS intensity of MB decreases with the increasing thrombin concentration. (b) The most prominent peak of MB, observed at $1622 \mathrm{~cm}^{-1}$, was compared upon addition of varying concentrations of thrombin. The limit of detection was estimated to be $100 \mathrm{pM}$. (c) The SERRS signal drop was proportional to the thrombin concentration and reached a saturation level at $1 \mu \mathrm{M}$ thrombin. However, the intensity was unaffected with increasing BSA concentrations up to $1 \mu \mathrm{M}$. Scale bar in a inset is $200 \mathrm{~nm}$. Note that the $y$-axis, i.e. normalized intensity $\left(\mathrm{I} / \mathrm{I}_{\mathrm{o}}\right)$, was plotted as a ratio of the measured intensity at each protein concentration over the intensity without thrombin in $1 \mathrm{x}$ thrombin- 
binding buffer solution. Data shown represents the mean, with standard deviation, of four separate measurements. 


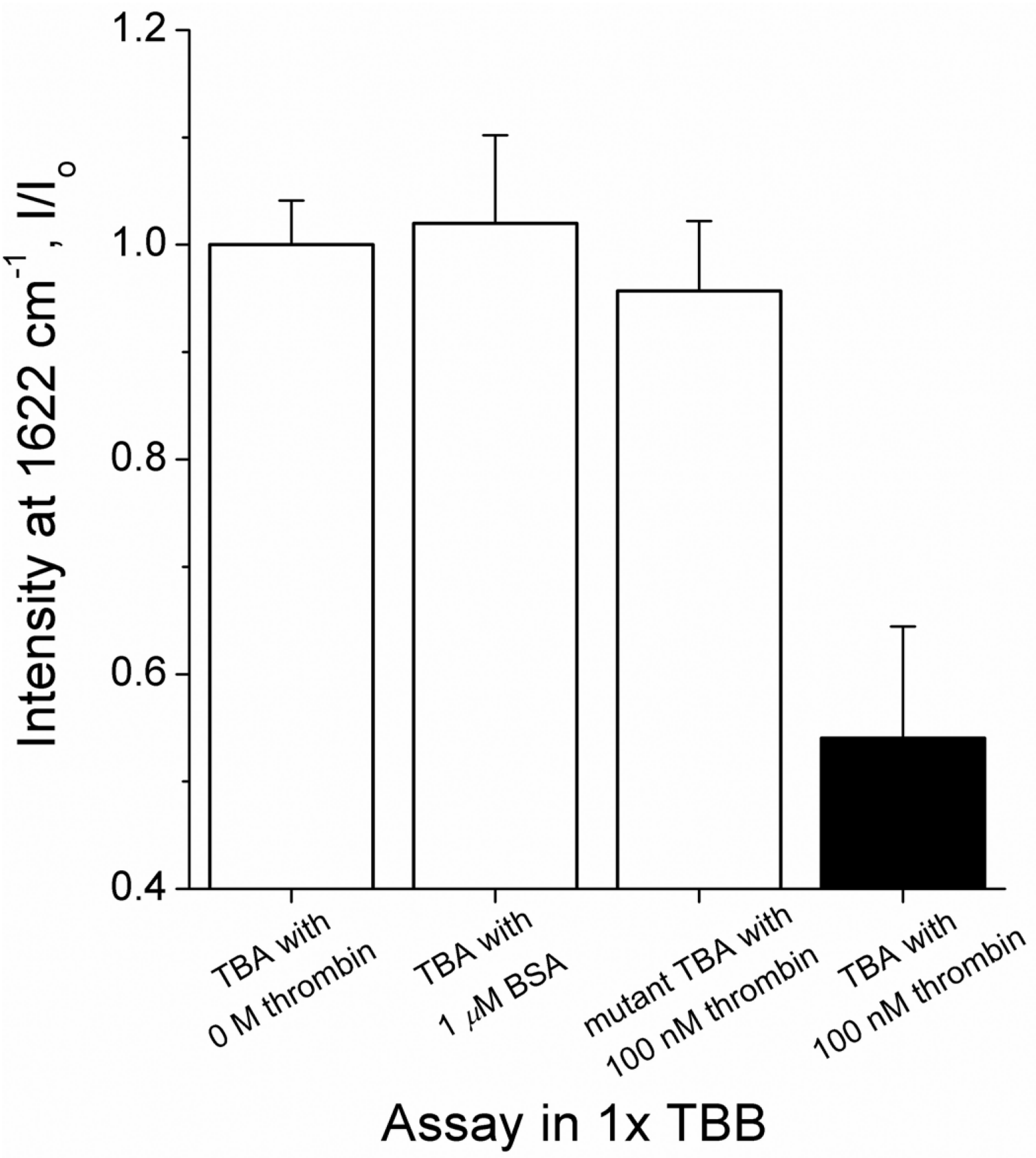

Figure 3.

Evaluation on the specificity of the developed method and the aptamer-based SERRS sensor. Assays were performed in 1x TBB. We did not observe any significant intensity change in SERRS signal from the TBA-GNP substrate in $1 \mu \mathrm{M}$ BSA or from the mutant TBA on GNP substrate in $100 \mathrm{nM}$ thrombin. In contrast, the SERRS signal drop was clearly distinguishable for the TBA-GNP substrate incubated with $100 \mathrm{nM}$ thrombin. Data shown represents the mean, with standard deviation, of four separate measurements. 


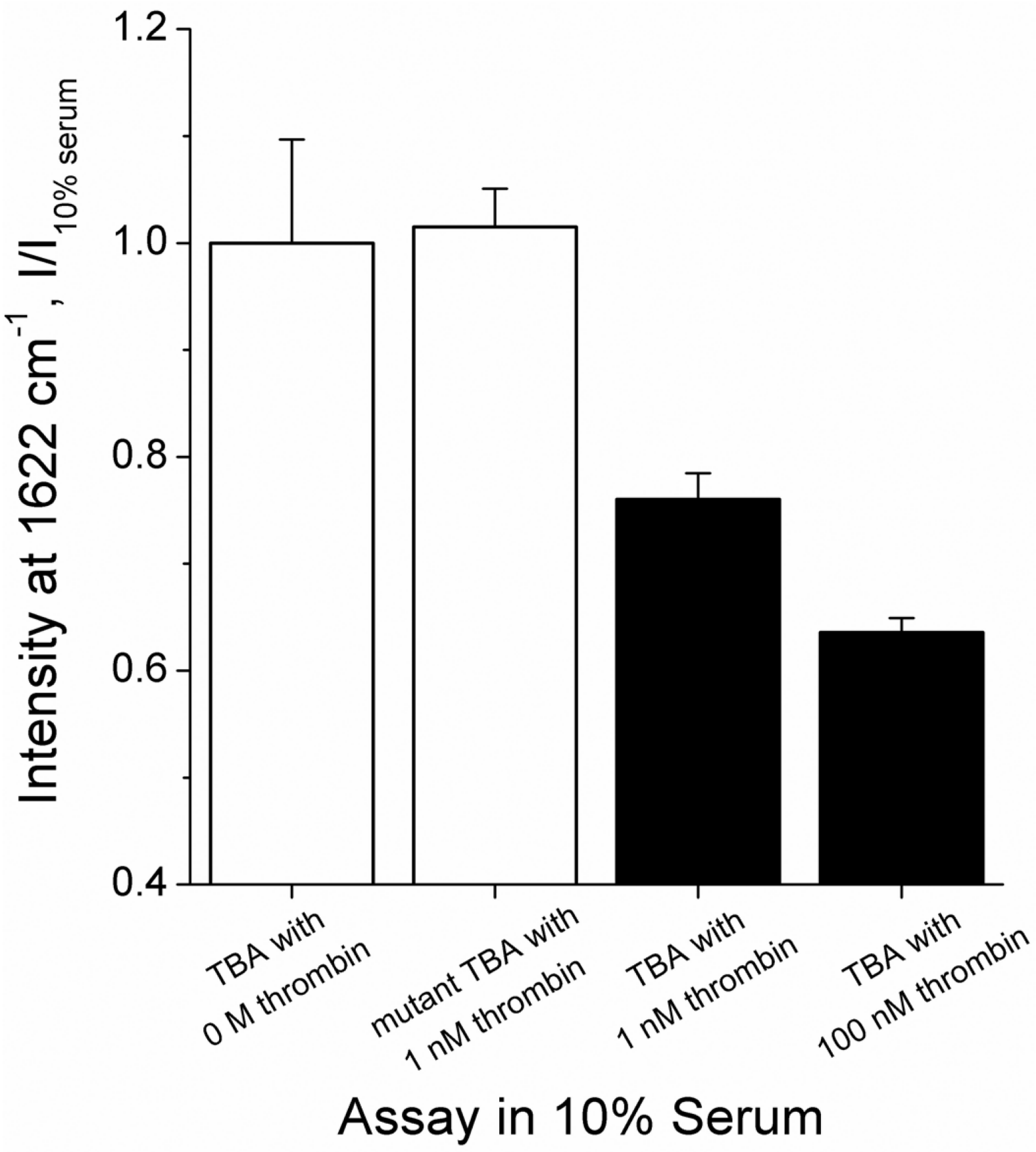

Figure 4.

Evaluation on the reliability of the developed method and the aptamer-based SERRS sensor. Assays were performed in 10\% fetal calf serum (v/v in 1x thrombin binding buffer). Based on a "serum baseline at $10 \%$ serum," the signal dropped to $\sim 75 \%$ with the addition of $1 \mathrm{nM}$ thrombin in $10 \%$ serum, while the signal dropped to a saturating level of $60 \%$ when the input thrombin was increased to $100 \mathrm{nM}$. Mutant TBA-GNP substrate was unaffected with the input of $1 \mathrm{nM}$ thrombin. Note that the $\mathrm{y}$-axis, i.e. normalized intensity $\left(\mathrm{I} / \mathrm{I}_{10 \%}\right.$ serum $)$, was plotted as a ratio of the measured intensity at each protein concentration over the intensity without thrombin in $10 \%$ serum. Data shown represents the mean, with standard deviation, of four separate measurements. 